\section{Evaluation Tool for the Assessment of Personal Protective Respiratory Equipment}

To the Editor-Respiratory protection is an important component of infection control measures designed to reduce the risk of exposure to respiratory pathogens. Well-documented cases of occupationally transmitted respiratory disease underscore the importance of well-designed and effectively implemented infection control programs that address respiratory protection. ${ }^{1,2}$ This is especially critical during the early stages of an outbreak of infection due to a novel pathogen, when effective pharmaceuticals may be unavailable or in short supply. The importance of adherence to respirator protocols was demonstrated during the outbreak of severe acute respiratory syndrome in 2002-2003, when many healthcare workers were infected and several died. ${ }^{3,4}$ Consistent and correct use of fit-tested N95 respirators and adherence to other infection control measures, such as standard and droplet precautions, will therefore likely be the most effective means of limiting occupational transmission of disease during the initial stage of outbreaks, including a pandemic event. ${ }^{5}$

Even though questions remain regarding the effectiveness of the various types of respiratory devices in protecting health care workers from infection, there is a growing consensus that N95 respirators will provide the best level of protection for many procedures performed by healthcare personnel. Currently, both the Centers for Disease Control and Prevention and the Occupational Safety and Health Administration recommend their use during a pandemic. ${ }^{5,6}$ However, this may change if additional information becomes available regarding the mode of transmission of a new pandemic pathogen (eg, a novel influenza virus). Beyond the issue of effectiveness, there is also a potential threat to workers resulting from their lack of consistent and correct use of respiratory protection, especially with respect to N95 respirators. Although data on worker compliance with proper N95 respirator use are sparse, the available information suggests that compliance is suboptimal at best. ${ }^{7} \mathrm{~A}$ recent report on healthcare workers' tolerance of N95 respirators found that 16 $(60 \%)$ of 27 subjects were unwilling to tolerate wearing the devices for an 8-hour period, even with intermittent breaks. ${ }^{8}$ The main reasons given for this included difficulties with communication, discomfort, and somatic complaints. Of particular interest was the finding that certain types of respirators were tolerated significantly better than others, and devices with exhalation valves were associated with greater tolerance levels. ${ }^{8}$

It would seem reasonable, therefore, that efforts be made to ensure that the devices chosen are those that are the most well tolerated among workers, especially given that healthcare facilities are increasingly stockpiling these devices to ensure an adequate supply during a pandemic event. Worker input about safety devices is an important part of an overall safety program, and indeed, it is a requirement for the selection of needle safety devices. ${ }^{9}$ Several evaluation tools have been developed to readily enable the collection of worker feedback regarding these types of devices. However, to our knowledge, similar evaluation tools are lacking for N95 respirators. To address this gap, we recently developed and pilot-tested an N95 respirator evaluation tool.

Three major criteria-fit/comfort, aesthetics, and somatic impact-were identified after a literature review of publications on compliance with instructions for the use of personal protective respiratory equipment and in consultation with experienced personnel from the hospital and emergency medical services sectors. Using a participatory action research framework, a team of 6 highly experienced, hospital-based registered nurses was recruited to develop and test an N95 evaluation tool. Points of evaluation that addressed each of the 3 major criteria were then identified. All of the nurses had previously been successfully fit-tested with their respective hospital's N95 devices and had worn N95 respirators. The study protocol was reviewed and approved by the Columbia University Institutional Review Board, and each participant provided signed informed consent. A 2-hour meeting was organized, in which each participating nurse received a packet that contained a disclosure and consent form, a list of the major evaluation criteria, and a set of 10 numbered N95 respirator models. All of the models were disposable or filtering face-piece respirators, and the models to be evaluated included cup, duckbill, and flat-fold styles. Several of the models were available to the nurses in different sizes, and some models had an exhalation valve feature. All of the N95 respirator models were approved by the National Institute for Occupational Safety and Health. ${ }^{10}$ Mirrors were available to aid the nurses in assessing the respirators' features. The nurses were asked to identify specific features relevant to each of the 3 criteria by using the various models to guide the discussion.

Once the N95 respirators were donned, it took the nurses an average of 5-10 minutes per respirator to discuss each model using the 3 key criteria. On the basis of this input, we constructed a final 20 -item tool with a 5-point rating scale (the numbers from 1 to 5 corresponded to a range of evaluations from "very poor" to "very good") (Figure).

The implementation of this assessment tool among workers who might be expected to wear these devices during work on the front lines of a pandemic or other respiratory disease outbreak may be a useful method of providing feedback to product selection or infection control committees. Given that N95 respirator intolerance might present a serious barrier to effective compliance with infection control protocols, the 


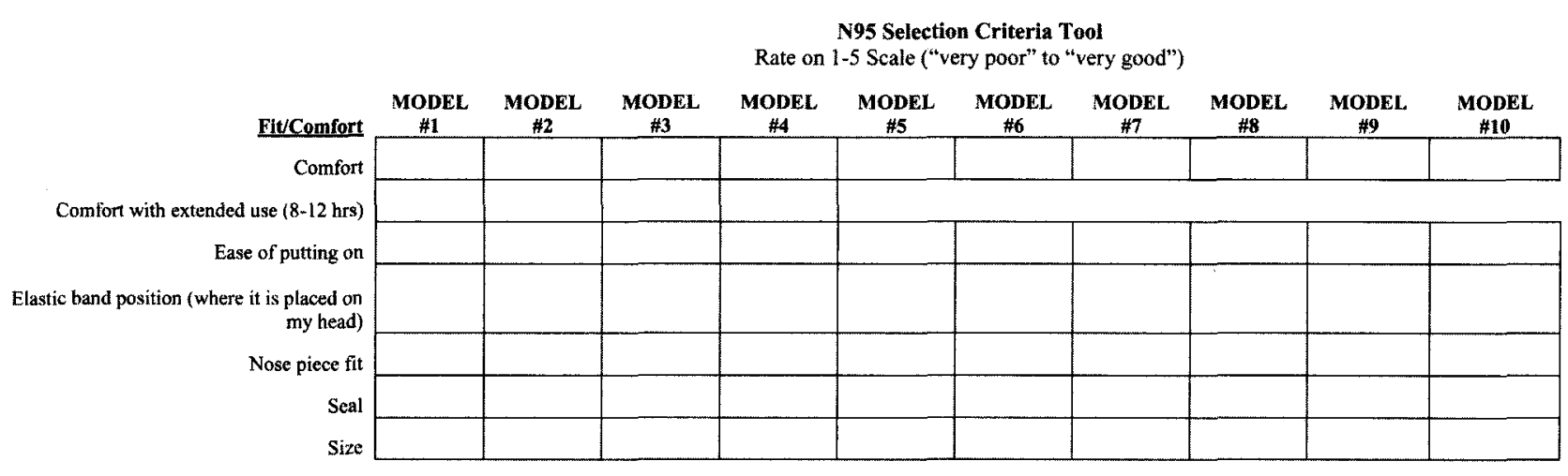

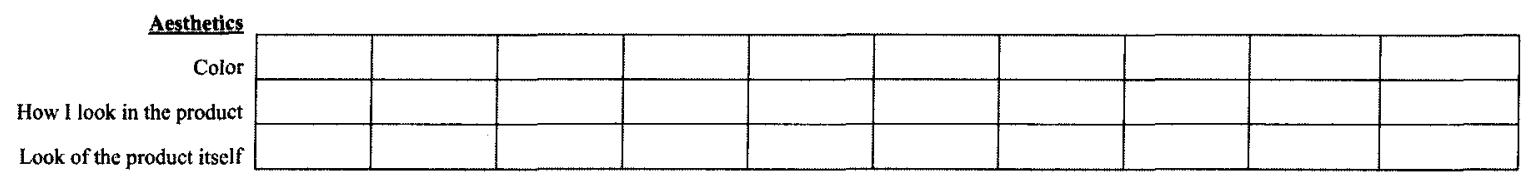

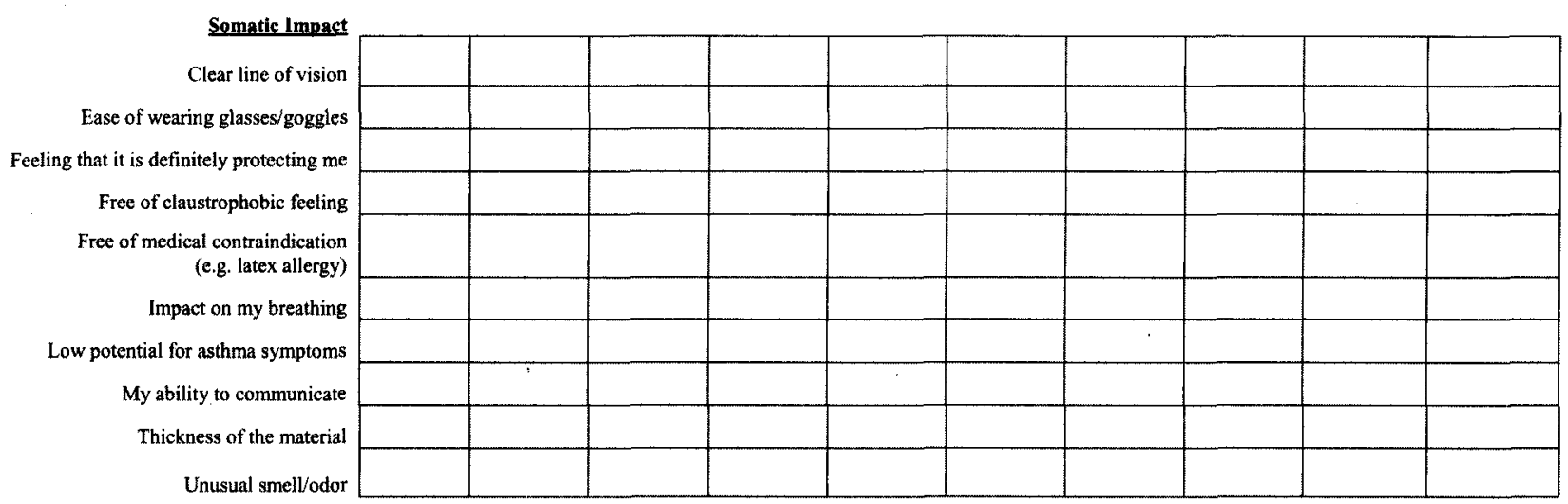

FIGURE. Evaluation tool used to assess N95 respirators in terms of 3 basic criteria.

consideration of these workers' input about the devices might help to improve compliance.

\section{ACKNOWLEDGMENTS}

We wish to thank Mr. James Wilcox for his invaluable collaboration on and contribution to the development of the evaluation tool. Financial support was provided by Avon Protection Systems (Baltimore, MD) to fund this study.

Financial support. This project was supported by Avon Protection Systems in conjunction with Columbia University Mailman School of Public Health Center for Public Health Preparedness.

\section{Robyn R. M. Gershon, MT, MHS, DrPH;} Julie M. Pearson, BA; Leah J. Westra, BA

From the Mailman School of Public Health, Columbia University, New York, New York.

Address reprint requests to Robyn R. M. Gershon, Mailman School of Public Health, Columbia University, 722 West 168th Street, Room 938, New York, NY10032 (rg405@columbia.edu).

The findings and conclusions in this report are those of the authors and do not necessarily represent the views of the funding agency.

Infect Control Hosp Epidemiol 2009; 30:716-718

(c) 2009 by The Society for Healthcare Epidemiology of America. All rights reserved. 0899-823X/2009/3007-0019\$15.00. DOI: 10.1086/600290

\section{REFERENCES}

1. Loeb M, McGeer A, Henry B, et al. SARS among critical care nurses. Emerg Infect Dis 2004; 10:251-255.

2. Teleman MD, Boudville IC, Heng BH, Zhu D, Leo YS. Factors associated with transmission of severe acute respiratory syndrome among healthcare workers in Singapore. Epidemiol Infect 2004; 132:797-803.

3. Centers for Disease Control and Prevention. Cluster of severe acute respiratory syndrome cases among protected health care workers-Toronto, April 2003. MMWR Morb Mortal Wkly Rep 2003; 52:433-436.

4. Dwosh HY, Hong HHL, Austgarden D, Herman S, Schabas R. Identification and containment of an outbreak of SARS in a community hospital. CMAJ 2003; 168:1415-1420.

5. Centers for Disease Control and Prevention, Pandemicflu.gov. Interim guidance on planning for the use of surgical masks and respirators in health care settings during an influenza pandemic. 2006. Available at: http://www.pandemicflu.gov/plan/healthcare/maskguidancehc.html. Accessed January 7, 2009.

6. United States Department of Labor, Occupational Safety and Health Administration. Pandemic influenza preparedness and response guidance for healthcare workers and healthcare employers. OSHA 3328-05R 2009. Available at: http://www.osha.gov/Publications/OSHA_pandemic_health.pdf. Accessed January 7, 2009.

7. Bryce E, Forrester L, Scharf S, Eshghpour M. What do healthcare workers think? A survey of facial protection equipment user preferences. J Hosp Infect 2008; 68:241-247. 
8. Radonovich LJ, Cheng J, Shenal BV, Hodgson M, Bender BS. Respirator tolerance in health care workers. JAMA 2009; 301:36-38.

9. United States Department of Labor, Occupational Safety and Health Administration. Occupational safety and health standards: bloodborne pathogens, final rule. 29 CFR $\$ 1910.1030$. Washington, DC: United States Department of Labor, Occupational Safety and Health Administration, 2001.

10. National Institute for Occupational Safety and Health. Respirator testing and certification (non-CBRN)-FY08. 2008. Available at: http://www .cdc.gov/niosh/nas/ppt/QUADCharts08/PP21_FY08_QC.htm. Accessed January $7,2009$.

\section{Did CA-MRSA Bacteremia Exist in Taiwanese Patients With End-Stage Renal Disease?}

To the Editor-In a recently published study, Lin et al. ${ }^{1}$ attempted to distinguish between the clinical characteristics of patients infected with community-associated methicillin-resistant Staphylococcus aureus (CA-MRSA) and those of patients infected with healthcare-associated (HA-MRSA). The study population consisted of patients who were receiving peritoneal dialysis or hemodialysis. This fact contradicts the present definition for CA-MRSA, because MRSA detected in persons with healthcare-associated risk factors, such as dialysis, within 1 year before onset of MRSA infection is not considered to be community acquired. ${ }^{2}$

One of the other criteria adopted by Lin and colleagues for identifying CA-MRSA and HA-MRSA was staphylococcal cassette chromosome (SCC) mec typing. They identified MRSA strains with SCCmec types IV or V as community acquired and MRSA strains with SCCmec types II or III as healthcare acquired. The designation of the source of MRSA acquisition by means of SCCmec typing may be misleading. In 2007, researchers at National Taiwan University Hospital (Taipei, Taiwan), the site of the study by Lin et al,, ${ }^{1}$ reported that SCCmec type III predominated during 1999-2004, whereas SCCmec types IV and V predominated during $2005 .^{3}$ Others have also reported changes in the predominant SCCmec types over time. ${ }^{4}$ Therefore, this use of SCCmec typing may not be an accurate method for distinguishing between HA-MRSA and CA-MRSA.

\section{ACKNOWLEDGMENTS}

Potential conflicts of interest. All authors report no conflicts of interest relevant to this letter.

Hung-Chin Tsai, MD, PhD; Cheng-Len Sy, MD; Yao-Shen Chen, MD
From the Section of Infectious Diseases, Department of Medicine, Kaohsiung Veterans General Hospital, Kaohsiung (H.-C.T., C.-L.S., Y.-S.C.), and the National Yang-Ming University, Taipei (H.-C.T., C.-L.S., Y.-S.C.), Taiwan

Address reprint requests to Hung-Chin Tsai, MD, Section of Infectious Diseases, Department of Medicine, Kaohsiung Veterans General Hospital, 386 Ta-Chung 1st Road, Kaohsiung 813, Taiwan (hctsai1011@yahoo.com tw).

All authors contributed equally to the composition of this letter. Infect Control Hosp Epidemiol 2009; 30:718-718

(C) 2009 by The Society for Healthcare Epidemiology of America. All rights reserved. 0899-823X/2009/3007-0020\$15.00. DOI: $10.1086 / 598238$

\section{REFERENCES}

1. Lin CC, Wang JL, Lin CY, et al. Methicillin-resistant Staphylococcus aureus bacteremia in patients with end-stage renal disease in Taiwan: distinguishing between community-associated and healthcare-associated strains. Infect Control Hosp Epidemiol 2009; 30:89-92.

2. Nathwani $D$, Morgan $M$, Masterton RG, et al. Guidelines for UK practice for the diagnosis and management of methicillin-resistant Staphylococcus aureus (MRSA) infections presenting in the community. I Antimicrob Chemother 2008; 61:976-994.

3. Huang YH, Tseng SP, Hu JM, Tsai JC, Hsueh PR, Teng LJ. Clonal spread of SCCmec type IV methicillin-resistant Staphylococcus aureus between community and hospital. Clin Microbiol Infect 2007; 13:717-724.

4. Wisplinghoff $\mathrm{H}$, Ewertz B, Wisplinghoff $\mathrm{S}$, et al. Molecular evolution of methicillin-resistant Staphylococcus aureus in the metropolitan area of Cologne, Germany, from 1984 to 1998. J Clin Microbiol 2005; 43:5445-5451.

\section{Reply to Tsai et al.}

To the Editor-We agree with Tsai et al. ${ }^{1}$ that staphylococcal cassette chromosome (SCC) mec typing may not be sensitive enough and specific enough to accurately classify methicillinresistant Staphylococcus aureus (MRSA) infections as either healthcare associated (HA) or community associated (CA). In addition to the different molecular epidemiologic characteristics of CA-MRSA strains in Taiwan, the evidence of continued spread of CA-MRSA strains into hospital settings ${ }^{2-4}$ and the detection of SCCmec type IV in a HA-MRSA strain, namely EMRA-15 (ST22-IV), which is endemic in many hospitals throughout the world, lead to occasional confusion regarding the definitions of CA-MRSA and HA-MRSA infections. ${ }^{5,6}$ However, molecular epidemiological definitions based on SCCmec typing and phylogenetic analyses of the MRSA isolates are still regarded as the most reliable means for distinguishing between HA-MRSA and CA-MRSA strains. ${ }^{5}$ In fact, MRSA strains carrying different SCCmec types are biologically different. The rationale for defining isolates carrying SCCmec types IV and V as CA-MRSA is based on the relatively small size of its genetic components, which facilitates the survival of CA-MRSA in the community setting. ${ }^{7,8}$ On the contrary, antibiotic selective pressure and cross-transmission in the nosocomial setting contribute to the survival of MRSA isolates 